\title{
Fluoxetine-enhanced autophagy ameliorates early brain injury via inhibition of NLRP3 inflammasome activation following subarachnoid hemorrhage in rats
}

Jian-ru Li ${ }^{1 \dagger}$, Hang-zhe $X u^{1+}$, Sheng Nie${ }^{1}$, Yu-cong Peng ${ }^{1}$, Lin-Feng Fan ${ }^{1}$, Zhi-jiang Wang ${ }^{1}$, Cheng Wu², Feng Yan ${ }^{1}$, Jing-yin Chen ${ }^{1}$, Chi Gu¹, Chun Wang ${ }^{1}$, Jing-sen Chen ${ }^{1}$, Lin Wang ${ }^{1}$ and Gao Chen ${ }^{1 *}$ (D)

\begin{abstract}
Background: The NLRP3 inflammasome is a multiprotein complex that regulates the innate immune inflammatory response by activating caspase-1 and subsequent IL-1 $\beta$ and IL-18. Fluoxetine has been shown to have the antiinflammatory properties in many disease models. However, the effects and mechanisms of these effects of fluoxetine in early brain injury after subarachnoid hemorrhage (SAH) have not been defined.

Methods: The SAH model was induced by an endovascular perforation in adult male Sprague-Dawley (SD) rats weighing 300-320 g. N-Ac-Tyr-Val-Ala-Asp-chloromethyl ketone (AC-WAD-CMK) was injected intraperitoneally $(5 \mathrm{mg} / \mathrm{kg}) 1 \mathrm{~h}$ after $\mathrm{SAH}$. Fluoxetine was administered via intravenous route $6 \mathrm{~h}$ after SAH. 3-Methyladenine (3-MA) was intracerebroventricularly injected 20 min before SAH. SAH grade, neurological function, brain water content, propidium iodide (PI) staining, western blot, double immunostaining, and transmission electron microscopy were performed.

Results: Expression of caspase-1 increased and peaked at $24 \mathrm{~h}$ after SAH. Caspase activation was along with the increased necrotic cells, which occurred mainly in neurons. Necrotic cell death of microglia and astrocyte were also found. Administration of AC-YVAD-CMK, a caspase-1 inhibitor, reduced the expression of IL-1 $\beta$ and IL-18 and the number of PI-positive cells, attenuated brain edema, and improved neurological function, which was also observed in fluoxetine-treated rats. Furthermore, fluoxetine treatment significantly decreased the expression of NLRP3 and cleaved caspase-1 and upregulated the expression of beclin-1, a marker for autophagy. Finally, the effects of fluoxetine in NLRP3 inflammasome activation were reversed by additional 3-MA administration.

Conclusions: Together, our present study indicated that NLRP3 inflammasome and caspase-1 activation play a deleterious role in early brain injury and fluoxetine mitigates NLRP3 inflammasome and caspase-1 activation through autophagy activation after SAH, providing a potential therapeutic agent for SAH treatment.
\end{abstract}

Keywords: Subarachnoid hemorrhage, NLRP3 inflammasome, Fluoxetine, Inflammation, Early brain injury, Autophagy

\footnotetext{
* Correspondence: d-chengao@zju.edu.cn

${ }^{\dagger}$ Equal contributors

'Department of Neurosurgery, The Second Affiliated Hospital of Zhejiang

University School of Medicine, Hangzhou, Zhejiang, China

Full list of author information is available at the end of the article
} 


\section{Background}

Subarachnoid hemorrhage $(\mathrm{SAH})$ refers to a consequence of bleeding within the subarachnoid space. Cerebral aneurysm rupture is the most frequent etiology of $\mathrm{SAH}$. Although SAH accounts for only 5\% of stroke cases, the mortality rate could be as high as $67 \%$ in the first few months [1, 2]. The significant mortality and morbidity may result from both the early brain injury and delayed cerebral ischemia [1]. Therapies targeted toward delayed cerebral ischemia have had limited success, which has led to a focus on mechanisms of early brain injury $[3,4]$. In the past few decades, many pathological mechanisms of early brain injury have been proposed. A ruptured aneurysm leads to many physiological derangements such as elevated intracranial pressure (ICP), decreased cerebral blood flow (CBF), and decreased cerebral perfusion pressure $(\mathrm{CCP})$. These events initiate various cascades of injuries such as inflammation, oxidative stress, blood brain barrier dysfunction, brain edema, and apoptosis [5, 6].

The inflammasome is a multiprotein complex that regulate the innate immune inflammatory response. Several inflammasomes, such as NLRP1, NLRP3, and AIM2, have been described in the central nervous system(CNS) and associated with brain injury $[7,8]$. Among them, NLRP3 inflammasome is the most studied one, especially in SAH $[9,10]$. Inflammasome activation triggers activation of caspase- 1 and mature of inflammatory cytokines, such as IL- $1 \beta$ and IL-18, activate the immune response eventually [11]. In addition, caspase- 1 activation also induces the regulated necrotic cell death, which is characterized by cell membrane rupture and subsequent inflammation [12]. Accumulating evidence have demonstrated that inhibiting NLRP3 inflammasome activation attenuates neuroinflammation and cell death in early brain injury and improves neurological function following $\mathrm{SAH}[9,10,13]$. Moreover, recent studies have uncovered that autophagy activation inhibited inflammasomes activation [14-16]. Fluoxetine, a selective serotonin reuptake inhibitor (SSRI), is widely used to treat depression, obsessive-compulsive disorder, bulimia, and panic disorder [17]. Recent studies have indicated that fluoxetine exhibits beneficial effects in neurological disorders, including ischemic stroke [18], brain injury [19], and spinal cord injury [20]. In fact, it is reported that fluoxetine induces the autophagy pathway through FK506 binding protein 51(FKBP51) [21]. Furthermore, fluoxetine inhibits NLRP3 inflammasome activation in depression [22]. However, the effect of fluoxetine on NLRP3 inflammasome activation and potential mechanisms in early brain injury after SAH remains unclear.

In the present study, we investigated the role of NLRP3 inflammasome and caspase-1 activation in early brain injury after $\mathrm{SAH}$ and the effect of fluoxetine on
NLRP3 inflammasome and caspase- 1 activation and the underlying possible mechanisms in early brain injury after SAH. Our findings indicated that caspase-1 activation was induced at the early stages of SAH and promoted necrotic cell death and expression of inflammatory cytokines (IL-1 $\beta$ and IL-18) in early brain injury after SAH. In addition, our study showed that fluoxetine reduces NLRP3 inflammasome and caspase-1 activation in early brain injury after SAH, at least partly, by activating autophagy, providing potential therapeutic interventions for $\mathrm{SAH}$.

\section{Methods \\ Animals}

Adult male Sprague-Dawley (SD) rats were purchased from Slac Laboratory Animal Company, Shanghai, China. The rats were housed in a controlled temperature and humidity conditions with a 12-h light/dark cycle.

\section{Study design}

The experiment was designed as follows (Additional file 1: Figure S1).

\section{Experiment 1}

Forty-eight rats were randomly divided into two groups: sham and SAH group. Western blot, propidium iodide (PI) staining, and transmission electron microscopy (TEM) were performed.

\section{Experiment 2}

Forty-three rats were randomly divided into the following four groups: sham, sham + AC-YVAD-CMK, SAH + vehicle, and SAH + AC-YVAD-CMK group.Western blot and PI staining were performed at $24 \mathrm{~h}$ after SAH. SAH grade and neurological scores were blindly assessed at the same time.

\section{Experiment 3}

Eighty-six rats were randomly assigned into the following four groups: sham, sham + Fluoxetine (Fluo), SAH + vehicle, and SAH + Fluo group. PI staining, double immunostaining, western blot, and brain water content were measured at $24 \mathrm{~h}$ after SAH. Neurological scores were also blindly assessed at the same time.

\section{Experiment 4}

Ninety-four rats were randomly assigned into the following five groups: sham, sham + 3-MA (Fluo), $\mathrm{SAH}+$ vehicle, $\mathrm{SAH}+$ Fluo group, and $\mathrm{SAH}+$ Fluo + 3-MA group. PI staining, western blot, and brain water content were measured at $24 \mathrm{~h}$ after SAH. Neurological scores were also blindly assessed at the same time. 


\section{SAH model}

The SAH model was induced by endovascular perforation as previously described [9], with some modification. Briefly, under pentobarbital $(50 \mathrm{mg} / \mathrm{kg})$ anesthesia, the left carotid artery and its branches were exposed and separated. Subsequently, the left external carotid artery (ECA) was cut, and a 4-0 monofilament suture was advanced into the internal carotid artery (ICA) through ECA until resistance was felt. Then, the suture was inserted further to puncture the vessel and induce SAH. The sham rats underwent the same procedure but without vessel puncture.

\section{Immunofluorescence staining}

Propidium iodide (PI; sigma) was dissolved in $0.9 \%$ $\mathrm{NaCl}$, and $10 \mathrm{mg} / \mathrm{kg}$ PI was administered intraperitoneally at $1 \mathrm{~h}$ prior to sacrifice. Brain coronal slices were obtained according to our previous protocol [23]. The slices were incubated at $4{ }^{\circ} \mathrm{C}$ over night with mouse monoclonal anti-neuronal nuclei (NeuN) (1:200, MAB377, Millipore), goat polyclonal anti-caspase-1 (1:200, sc-22165, Santa Cruz), and rabbit monoclonal anti-LC3 (1:200, 3868\#, Cell signaling Technology) followed by incubation with appropriate secondary antibody (1:200, Jackson ImmunoResearch). Double immunostaining was performed to identify the relation between pyroptosis and autophagy. The primary antibodies that were used were the rabbit monoclonal anti-LC3 (1:200, 3868\#, Cell signaling Technology) and goat polyclonal anti-caspase-1 (1:200, sc-22165, Santa Cruz). The secondary antibodies that were used were rhodamine-conjugated donkey anti-rabbit antibody (1:200, Jackson ImmunoResearch) and fluorescein isothiocyanate-labeled donkey anti-goat antibody (1:200, Jackson ImmunoResearch). The sections were blindly observed using a fluorescence microscope.

\section{Transmission electron microscopy}

Rats were sacrificed under deep anesthesia by cardiac perfusion with $0.9 \%$ saline and $4 \%$ paraformaldehyde in $0.1 \mathrm{M}$ PBS. We obtained the $1-\mathrm{mm}^{3}$ brain slices from the left parietal cortex; samples were then transferred into $2.5 \%$ glutaraldehyde and kept overnight at $4{ }^{\circ} \mathrm{C}$. Samples were rinsed several times with buffer and fixed with $1 \%$ osmium tetroxide for $1 \mathrm{~h}$. After rinsing again with the distilled water several times, the samples were dehydrated with a series of graded ethanol. Next, infiltration was implemented using a solution of propylene oxide and resin (1:1). Samples were then embedded in resin the next day. $100 \mathrm{~nm}$ sections were cut and $4 \%$ uranyl acetate $(20 \mathrm{~min})$ and $0.5 \%$ lead citrate $(5 \mathrm{~min})$ were used for staining. A transmission electron microscope was used to examine the cortex ultrastructure.

\section{Drug administration}

N-Ac-Tyr-Val-Ala-Asp-chloromethyl ketone (Ac-YVADCMK, Cayman Chemical Company) was dissolved in DMSO and was then diluted in PBS, and injected intraperitoneally $(5 \mathrm{mg} / \mathrm{kg}) 1 \mathrm{~h}$ after the induction of $\mathrm{SAH}$ as previously described [24]. Fluoxetine was purchased from Selleckchem and dissolved in $0.9 \%$ $\mathrm{NaCl}$. Fluoxetine $(10 \mathrm{mg} / \mathrm{kg})$ or vehicle was administered intravenously at $6 \mathrm{~h}$ after $\mathrm{SAH}$ induction as previously described [25]. For the mechanism study, rats were fastened to a stereotactic frame and treated with $5-\mu \mathrm{l}$ intracerebral ventricular injection of autophagy inhibitor 3-methyladenine (3-MA; $400 \mathrm{nmol}$ ) $20 \mathrm{~min}$ before $\mathrm{SAH}$ onset. 3-MA was purchased from Sigma-Aldrich. Coordinates for intracerebral ventricular injection were $0.8 \mathrm{~mm}$ posterior to anterior bregma, $1.0 \mathrm{~mm}$ mid to lateral, and $3.8 \mathrm{~mm}$ dorsal to ventral. The dose of 3-MA and the coordinates of intracerebral ventricular injection were chosen according to the procedure used in our previous study [26]. After the injection, the needle was left in the site for $5 \mathrm{~min}$ before removal and the hole was filled with bone wax.

\section{SAH grade}

The degree of SAH was blindly evaluated as described by Sugawara et al. [27]. In brief, the basal cistern was divided into six parts. Based on the amount of blood in the subarachnoid space, each part was given a score from 0 to 3 and $\mathrm{SAH}$ grade was calculated as the sum of each score.

\section{Neurological scores}

The modified Garcia score system was blindly performed to assess the neurological function after SAH [27]. The neurological scores were calculated from a sum of scores from six categories as follows: spontaneous activity, spontaneous movements of all limbs, movements of forelimbs, climbing the wall of the cage, reaction to touch on both sides of the trunk, and response to vibrissae touch. The maximum obtainable score is 18 .

\section{Brain water content}

Rats were decapitated under deep pentobarbital anesthesia. Subsequently, the left hemispheres were removed and immediately weighed to obtain the wet weight and then dried at $100{ }^{\circ} \mathrm{C}$ for $48 \mathrm{~h}$ to obtain the dry weight as previously described [9]. The brain water content was calculated as (wet weight-dry weight)/wet weight $\times 100 \%$.

\section{Western blot}

Western blot was performed according to our previously protocols [28]. Briefly, the brain tissue was homogenized and centrifuged. The detergent compatible protein assay 
kit (Bio-Rad, Hercules, CA, USA) was used to determine the protein content. Equal amounts of protein $(60 \mu \mathrm{g})$ were loaded into the wells of the SDS-PAGE gel. After electrophoresis, the protein was transferred to a nitrocellulose membrane. The membrane was blocked for $2 \mathrm{~h}$ using a nonfat dry milk buffer and incubated overnight at $4{ }^{\circ} \mathrm{C}$ with the primary antibodies. The following primary antibodies were used: rabbit polyclonal anti-beclin 1 (1:800, ab62557, Abcam), rabbit monoclonal antiNLRP3(1:1000, ab210491, Abcam), goat polyclonal anti-caspase-1 (1:2000, sc-22165, Santa Cruz), rabbit polyclonal anti-IL-1ß (1:200, ab7973, Abcam), rabbit polyclonal anti-IL-18 (1:1000, cst\# 9272, Cell Signaling Technology), and $\beta$-actin (1:4000, sc-47778Santa Cruz). The membrane was incubated for $1 \mathrm{~h}$ with secondary antibodies (horseradish peroxidase-conjugated) at room temperature. The membrane was exposed to X-ray film, and band densities were analyzed using Image J software. To facilitate comparisons between groups, band density values were normalized to the mean value for the sham group.

\section{Statistical analysis}

The average band density for the control group was set at 1.0, and all band density values were normalized by the average value of the control group. Statistical analyses for two sets of data were performed using Student's $t$ test. A one-way analysis of variance (ANOVA) followed by a Tukey's multiple comparisons test was used to analyze data for multiple groups. The comparisons of behavior and activity scores were analyzed using the Mann-Whitney $U$ test. The data were represented as the mean \pm SD. $P<0.05$ was used for statistical significance.

\section{Results}

Mortality

The physiological parameters, such as the mean blood pressure and blood gases, were observed, and these parameters were not significantly different between the groups (data not shown). None of the rats died in sham, sham + AC-YVAD-CMK, sham + Fluo, and sham + 3MA group. In experiment 1 , the mortality of the $\mathrm{SAH}$ group was $31.6 \%$ (12 of 38 rats). In experiment 2 , the mortalities were $30.8 \%$ ( 4 of 13 rats) and 25\% (3 of 12 rats) in the $\mathrm{SAH}+$ vehicle group and the $\mathrm{SAH}+\mathrm{AC}$ YVAD-CMK group, respectively. In experiment 3 , the $\mathrm{SAH}+$ vehicle group exhibited $33.3 \%$ (9 of 27 rats) mortality, whereas $21.7 \%$ (5 of 23 rats) mortality occurred in $\mathrm{SAH}+$ fluoxetine group. In experiment 4 , the mortality reached $30.4 \%$ (7 of 23 rats), $25 \%$ (5 of 20 rats) in the $\mathrm{SAH}+$ fluoxetine group, and $28.6 \%$ (6 of 21 rats) in the $\mathrm{SAH}+$ fluoxetine + 3-MA group. There were no significant differences in mortality between the groups (data not shown).
Caspase-1 activation occurred in the early stages and accompanied with increased neural necrotic cell death after SAH

Western blot results showed that caspase- 1 expression levels were significantly increased at $12 \mathrm{~h}$ and peaked at $24 \mathrm{~h}$ after SAH $(P<0.01$; Fig. 1a). After peaking, caspase- 1 expression levels were declined, but were still high at $72 \mathrm{~h}$ post-SAH when compared with the sham group $(p<0.05$; Fig. 1a). In addition, compared with the sham group, the number of PI-positive cells markedly increased in the SAH group at $24 \mathrm{~h}$ after SAH $(P<0.01$; Fig. $1 b)$. Double immunostaining showed that most of the necrotic cell death was found in neurons, while some were found in microglia and astrocyte (Fig. 2a). Meanwhile, we found that cleaved caspase-1 expression in the necrotic neural cell (Fig. 2b). We further confirmed this caspase- 1 antibody did not detect the pro-form of caspase-1 (Additional file 2: Figure S2). Meanwhile, we identified the ultrastructural features of necrotic cells using electron microscopy. As represented in Fig. $2 \mathrm{c}$, neurons in the sham cortex appeared normal with a nucleus, mitochondria, and cytomembranes. Neurons from $\mathrm{SAH}$ rats showed a fragmented nucleus (black asterisk), swollen mitochondria (red asterisk), disrupted cytomembranes (white arrow), and damaged organelles (black arrow). Interestingly, we observed double-membrane autophagosome in neurons from SAH rats (red arrow).

\section{Caspase-1 activation contributes to early brain injury after SAH}

Western blot results showed that IL-1ß and IL-18 levels were significantly elevated when compared with the sham group at $24 \mathrm{~h}$ after $\mathrm{SAH}(P<0.01$, Fig. $3 \mathrm{a}, \mathrm{b})$. The protein levels of IL-1ß and IL-18 were significantly decreased by Ac-YVAD-CMK administration $(P<0.01$; Fig. 3a, b). PI staining showed that PI-positive neural cells were significantly increased in the SAH group when compared with the sham group $(P<0.01$; Fig. 3c, d). Administration of Ac-YVAD-CMK resulted in a significant reduction of PIpositive neural cells compared with the $\mathrm{SAH}$ group $(P<0.01$; Fig. 3c, d). To further confirm the protective effect of Ac-YVAD-CMK at the macroscopic level, the neurological functions were measured. Consistent with the above results, severe neurological deficits were found in the SAH group compared with the sham group $(P<0.05$; Fig. 3e), which were improved by AC-YVADCMK administration $(P<0.05$; Fig. $3 e)$. In addition, there was no significant difference between the SAH group and the SAH + AC-YVAD-CMK group $(P>0.05$; Fig. $3 f)$.

\section{Fluoxetine attenuates NLRP3 inflammation activation and brain edema and improved neurological function at $24 \mathrm{~h}$ after SAH}

Western blot analysis revealed the expression of NLRP3, cleaved caspase-1, IL-1 $\beta$ and IL-18 were significantly 


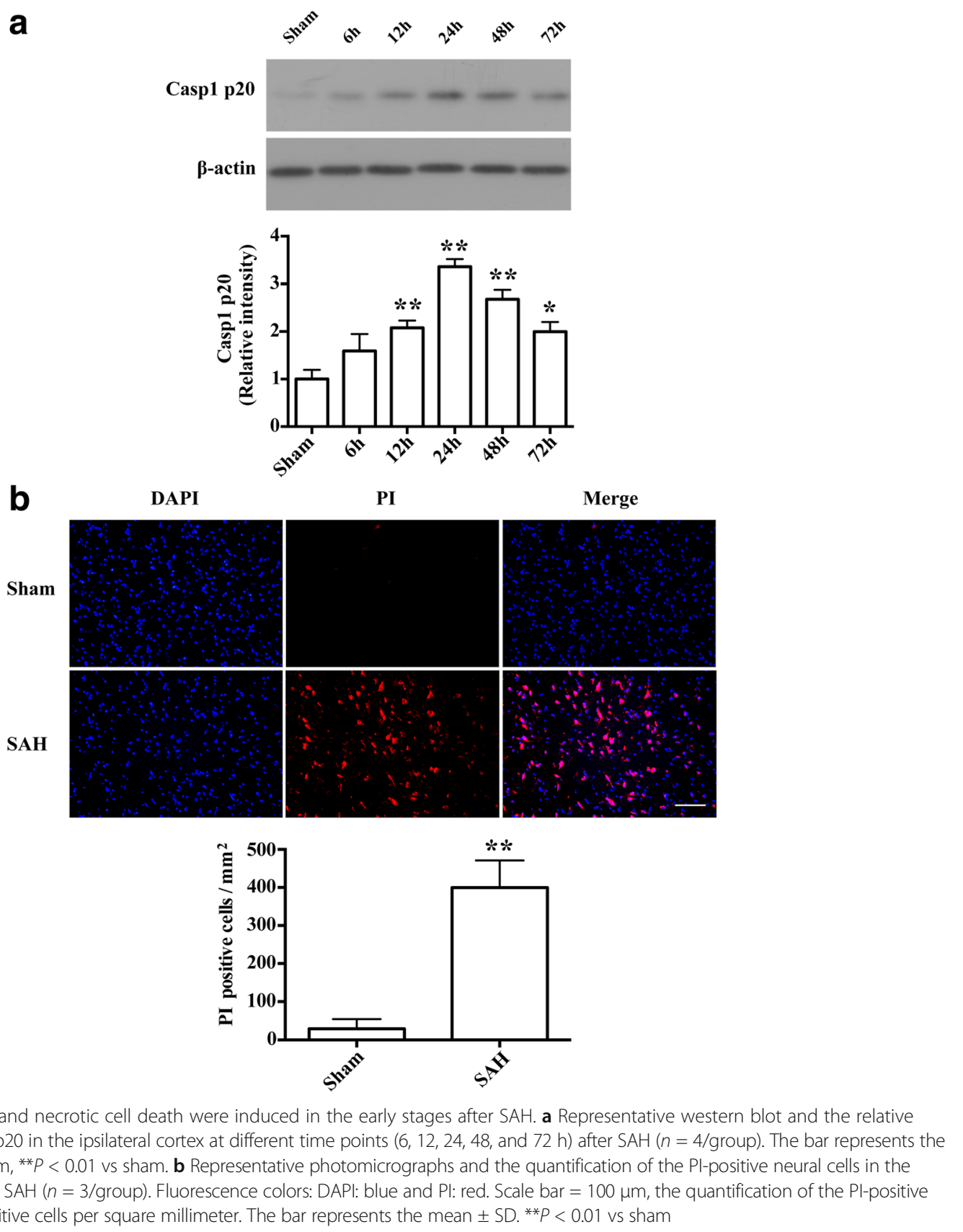

increased compared with the sham rats $(P<0.01$; Fig. 4a). Fluoxetine administration significantly reduced expression of NLRP3 $(P<0.05$; Fig. 4a), cleaved caspase$1(P<0.01$; Fig. 4a), IL-1 $\beta(P<0.01$; Fig. $4 a)$, and IL-18 $(P<0.01$; Fig. 4a). Meanwhile, fluoxetine treatment significantly reduced the number of PI-positive neural cells compared to the SAH + vehicle group $(P<0.01$; Fig. $4 \mathrm{~b}, \mathrm{c})$. With regard to brain edema in the ipsilateral hemisphere, fluoxetine significantly reduced the SAHinduced brain edema $(P<0.01$; Fig. $4 \mathrm{~d})$. At $24 \mathrm{~h}$ after $\mathrm{SAH}$, fluoxetine administration significantly improved the neurological function compared with the SAH + vehicle group $(P<0.05$; Fig. 4e).

\section{Neuroprotective effects of fluoxetine on NLRP3} inflammasome involve autophagy activation after $\mathrm{SAH}$ Double immunostaining showed that LC3 expressions were observed in PI-positive neural cells (Fig. 5a) and co-localized with cleaved caspase- 1 in the cortex at $24 \mathrm{~h}$ after SAH (Fig. 5b). To further evaluate the autophagy activation, we examined the levels of the autophagyrelated protein, becline 1 . Western blot results showed 


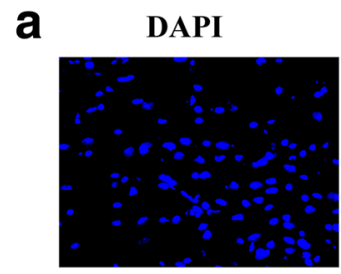

DAPI

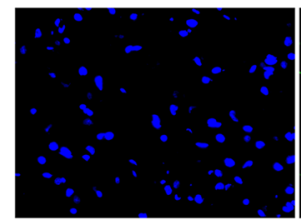

DAPI

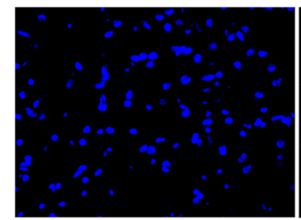

b

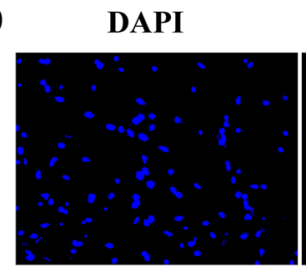

C

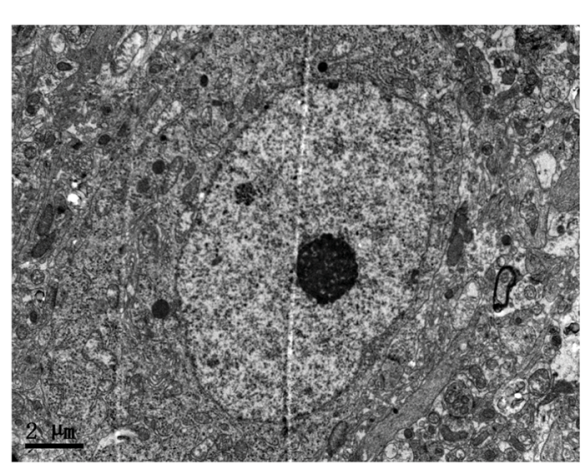

PI

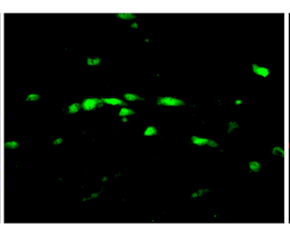

Iba-1

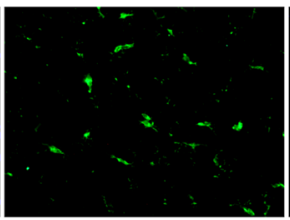

GFAP

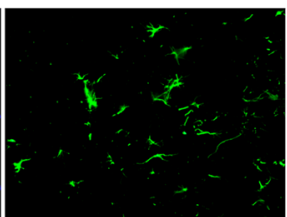

Caspase-1

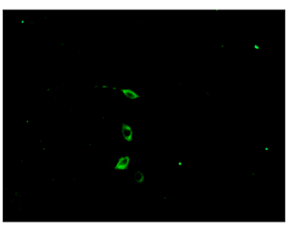

Sham

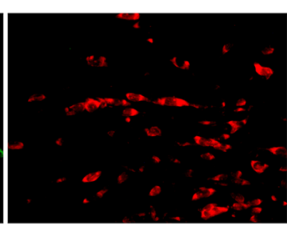

PI

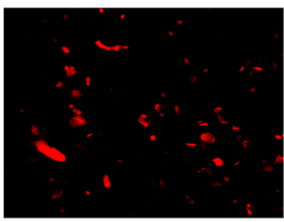

PI

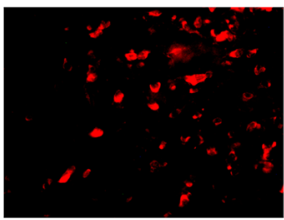

PI

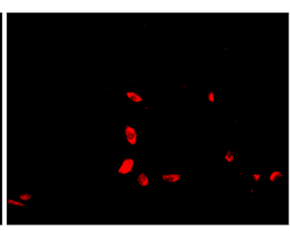

SAH

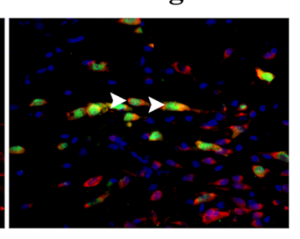

Merge

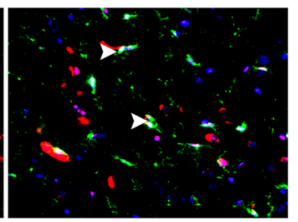

Merge

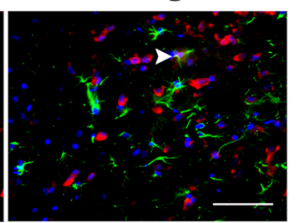

Merge
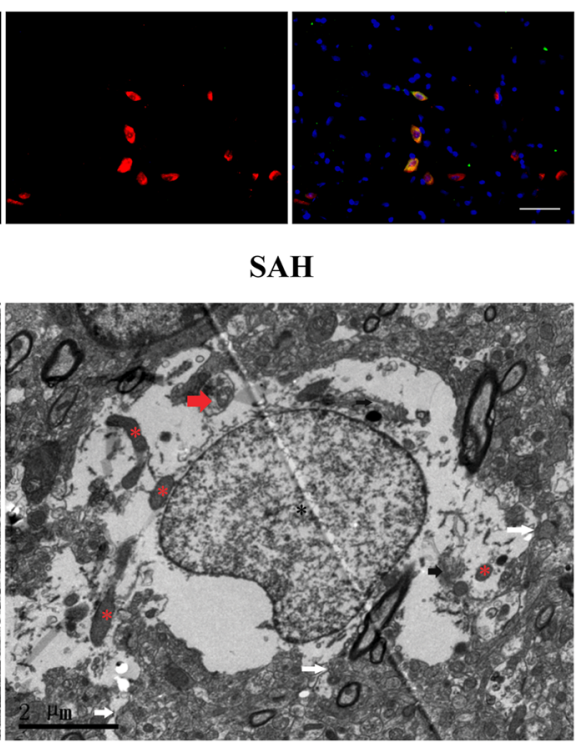

Fig. 2 Double staining for PI with NeuN (neuron marker), Iba-1 (microglia marker), GFAP(astrocyte marker), and caspase-1, and ultrastructural changes of necrotic cells. a Double staining for PI with NeuN (neuron marker), Iba-1(microglia marker), and GFAP(astrocyte marker). Scale bar $=50 \mu \mathrm{m}$. b Double staining for PI with caspase- 1 in the ipsilateral cortex at $24 \mathrm{~h}$ after SAH. Scale bar $=50 \mu \mathrm{m}$. c Ultrastructural changes of necrotic cells at $24 \mathrm{~h}$ after SAH. Neurons in the left panel appeared normal with nuclei, mitochondria, and cytomembranes. Neurons in the right panel showed fragmented nuclei (black asterisk), swollen mitochondria (red asterisk), disrupted cytomembranes (white arrow), damaged organelles (white arrow), and double-membrane autophagosomes (red arrow). Scale bar $=2 \mu \mathrm{m}$

that fluoxetine administration upregulated becline 1 levels $(P<0.05$; Fig. 5c), indicating autophagy activation.

Autophagy inhibitor 3-MA blocks the effects of fluoxetine on NLRP3 inflammasome activation and brain edema, aggravating neurological deficits after SAH

To confirm autophagy activation in the neuroprotective effect of fluoxetine, autophagy inhibitor 3-MA was used.
Western blot results showed that co-administration of fluoxetine and 3-MA significantly increased the levels of NLRP3, cleaved caspase-1, mature IL-1ß, and IL-18 compared with the SAH + fluoxetine group $(P<0.05$; Fig. 6a, b). Notably, immunostaining results showed that co-administration of fluoxetine and 3-MA significantly increased the number of PI-positive neural cells compared to the SAH + fluoxetine rats $(P<0.01$, Fig. 7a, b). 


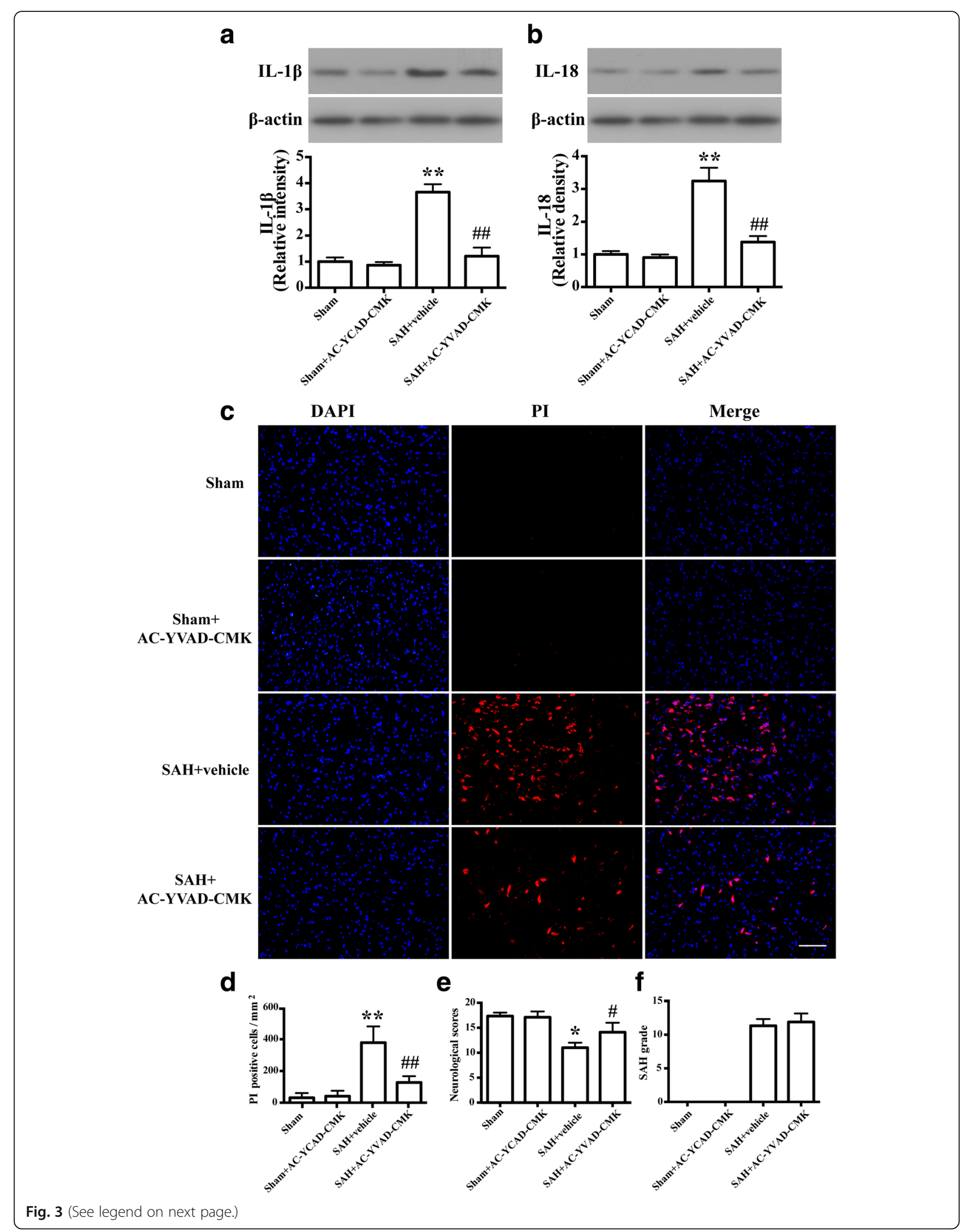


(See figure on previous page.)

Fig. 3 Caspase-1 activation contributes to early brain injury after SAH. a Representative western blots and densitometric analyses showing levels of IL-1ß. The bar represents the mean \pm SD. ${ }^{*} P<0.01$ vs sham. ${ }^{\# \#} P<0.01$ vs vehicle control. b Representative western blots and densitometric analyses showing levels of IL-18 ( $n=6 /$ group). The bar represents the mean $\pm S D$. ${ }^{* *} P<0.01$ vs sham. ${ }^{\# \#} P<0.01$ vs vehicle control. c Representative photomicrographs of PI staining of neural cells in different groups at $24 \mathrm{~h}$ after SAH ( $n=3$ /group). Fluorescence colors: DAPI: blue and Pl: red. Scale bar $=100 \mu \mathrm{m}$. $\mathbf{d}$ The quantification of the PI-positive neural cells. The quantification of the PI-positive neural cells expressed as positive cells per square millimeter. The bar represents the mean \pm SD. ${ }^{* *} P<0.01$ vs sham, ${ }^{\# \#} P<0.01$ vs vehicle control. e Quantification of neurological scores $(n=9)$. The bars represent the mean with interquartile range. ${ }^{*} P<0.05$ vs sham. ${ }^{\#} P<0.05$ vs vehicle control. f Quantification of SAH severity $(n=9)$. The bars represent the mean \pm SD. No significant difference was observed between vehicle and SAH+AC-YVAD-CMK group

With regard to brain edema in the ipsilateral hemisphere, co-administration of fluoxetine and 3-MA significantly augmented the increased brain edema $(P<0.05 ;$ Fig. $7 \mathrm{c})$ and worsened the neurological function $(P<0.05$; Fig. $7 \mathrm{~d})$ compared with the SAH + fluoxetine group.

\section{Discussion}

The present study provided the evidence that caspase-1 activation-induced necrotic neural cell death and inflammatory cytokines IL-1 $\beta$ and IL-18 contributed to the early brain injury after SAH. In addition, our report found that fluoxetine attenuated NLRP3 inflammasome and caspase- 1 activation and subsequent inflammatory cytokines expression as well as necrotic cell death, reduced the brain edema, and improved neurological deficits. Moreover, autophagy activation was involved in these beneficial effects of fluoxetine, which were abolished by autophagy inhibitor 3-MA. Taken together, our findings suggested that fluoxetine administration, targeting NLRP3 inflammasome and caspase-1 activation, might be an effective therapeutic strategy after SAH.

Caspase-1 is an inflammatory caspase that is well known for the function of cleaving the preforms of inflammatory cytokines IL-1 $\beta$ and IL-18 into their active forms [29]. Both IL-1 $\beta$ and IL-18 are the important inflammatory mediator in early brain injury after SAH. These inflammatory cytokines induced matrix metalloproteinase (MMP)-9 expression and disruption of the blood brain barrier (BBB), aggravating brain edema and neurological deficits after SAH [24]. Clinical studies have demonstrated that caspase- 1 and subsequent inflammatory cytokines elevated in cerebrospinal fluid (CSF) of patients with $\mathrm{SAH}[30,31]$. In the current study, we found that cleaved caspase- 1 was increased and peaked at $24 \mathrm{~h}$ after SAH. Besides, increasing studies indicated that caspase- 1 also induced the necrotic cell death by cleaving adermin-D (GSDMD), which lyses the liposomes and forms the pores on the membranes [32-34]. Our study indicated that caspase- 1 activation was accompanied with increased necrotic neural cells. In addition, most of these necrotic neural cells were neurons, which is consistent with previous studies [35]. And some of them were microglia and astrocytes. Additionally, the ultrastructural features of necrotic cell death were identified by using a transmission electron microscope. DNA fragmentation, swollen mitochondria, damaged cytoplasmic organelles, cytoplasmic hypervacuolization, and cytomembrane rupture were observed. Intriguingly, autophagic vacuoles were also observed, indicating that autophagy may be involved in the pathology of caspase-1-induced necrotic cell death. These features are consistent with previous studies [36, 37]. Caspase-1 inhibitor AC-YVAD-CMK downregulated the expression of mature IL-1 $\beta$ and IL-18, reduced the number of necrotic cell death, and improved neurological function in SAH rats. Taken together, our study indicated that caspase-1 activation contributes to the early brain injury after SAH.

Caspase-1 activation requires a multiprotein platform, namely inflammasome. To date, NLRP1b, NLRP3, and NLRC4, as well as the cytosolic DNA sensor absent from in melanoma2(AIM2), are well-defined inflammasomes [38]. Our previous studies demonstrated NLRP3 inflammasome contribute to the neuroinflammation in early brain injury after SAH $[9$, 14]. A recent clinical study demonstrated NLRP3 inflammasome and caspase- 1 were elevated in CSF of $\mathrm{SAH}$ patients and associated with functional outcome of these patients [30]. Therapies targeting NLRP3 inflammasome activation attenuated early brain injury and improved neurological function after $\mathrm{SAH}$ in rats $[9,10,39]$. Traditionally, fluoxetine is a common treatment for major depression due to its safer profile, greater tolerability, and fewer side effects. Recently, the protective pharmacological effects of fluoxetine have been demonstrated in clinical practice in the treatment of neurological diseases, such as ischemic [18], Alzheimer's disease [40], and traumatic brain injury [41]. Emerging evidence suggests that fluoxetine has anti-inflammatory properties [42, 43]. But the precise mechanism is uncertain. In our present study, fluoxetine was found to reduce the expression of NLRP3, cleaved caspase-1, IL-1 3 , and IL18 , alleviate the neural necrotic cell death and brain 


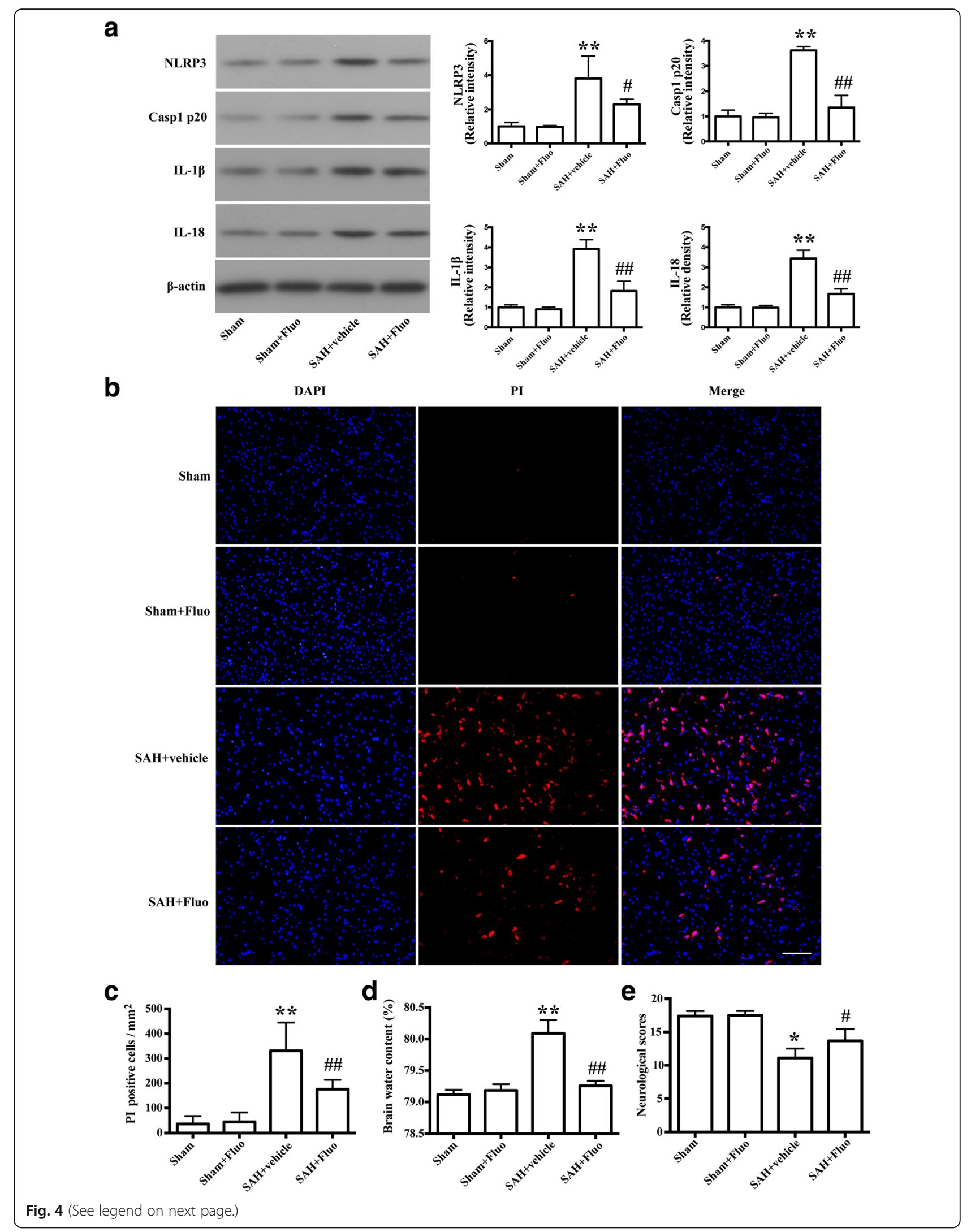


(See figure on previous page.)

Fig. 4 Fluoxetine reduced expression of NLRP3, caspase-1 p20, IL-1B, and IL-18, decreased the numbers of necrotic cells and brain edema, and improved the neurological scores. a Representative Western blots and densitometric analyses of levels of NLRP3, caspase-1 p20, IL-1B, and IL-18. The bar represents the mean \pm SD. ${ }^{*} P<0.01$ vs sham. ${ }^{\#} P<0.05$ vs vehicle control, ${ }^{\# \#} P<0.01$ vs vehicle control. b Representative photomicrographs of Pl-positive neural cells in different groups at $24 \mathrm{~h}$ after SAH ( $n=3 /$ group). Fluorescence colors: DAPI: blue and PI: red. Scale bar $=100 \mu \mathrm{m}$. $\mathbf{c}$ The quantification of the PI-positive neural cells. The quantification of the PI-positive neural cells expressed as positive cells per square millimeter. The bar represents the mean \pm SD. ${ }^{* *} P<0.01$ vs sham, ${ }^{\# \#} P<0.01$ vs vehicle control. d Quantification of brain water content $(n=6)$. The bars represent the mean \pm SD. ${ }^{*} P<0.01$ vs sham control,

${ }^{\# \#} P<0.01$ vs vehicle control. e Quantification of neurological scores $(n=18)$. The bars represent the mean with interquartile range. ${ }^{*} P<0.05$ vs sham control, $\# P<0.05$ vs vehicle control

edema, and improve the neurological function after SAH. These data indicated that fluoxetine alleviates early brain injury after SAH through the inhibition of NLRP3 inflammasome activation.

Autophagy is an intracellular process for cellular homeostasis and recycling of damaged organelles and proteins, as well as the destruction of intracellular pathogens [44]. Activated autophagy pathway has been demonstrated in experimental SAH [26, 45, 46]. More and more studies have demonstrated the regulatory roles of autophagy in inflammasome activation. The loss of proteins that are essential for autophagy activation, such as Atg16L1 and Atg7, resulting in caspase-1 activation as well as increased production of IL-1 $\beta$ and IL-18 in macrophages $[47,48]$. In addition, autophagy can also negatively regulate inflammasome activation through removing damaged mitochondria to preventing the release of mtROS and mtDNA into the cytoplasm, and ultimately limiting inflammasome assembly [49, 50]. Additionally, assembled inflammasomes can be degraded by autophagosomes through the autophagic protein $\mathrm{p} 62$ $[51,52]$. Fluoxetine upregulated the expression of
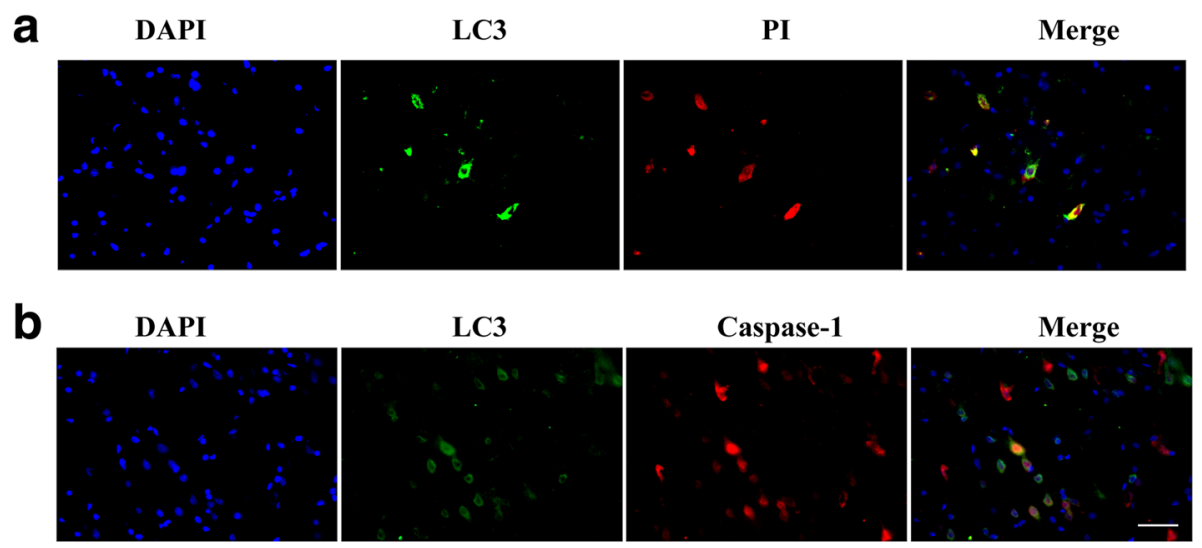

C

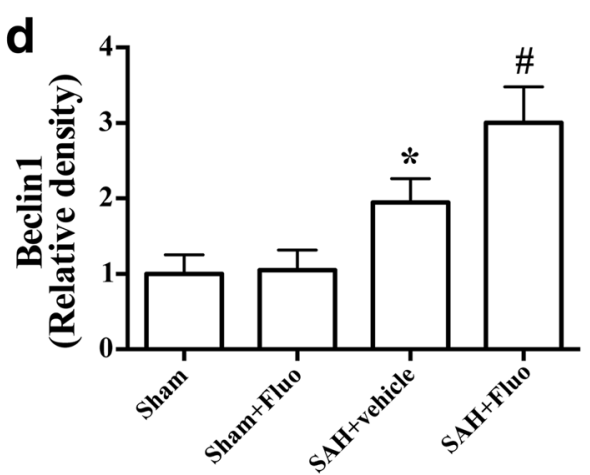

Fig. 5 Autophagy activation was involved in the protective effect of fluoxetine on NLRP3 inflammasome activation. a Double fluorescence of PI staining (red) and cells with autophagy marker LC3 (green). b Representative micrographs showing double immunofluorescence with caspase-1 (red) and LC3 (green). Scale bar $=50 \mu \mathrm{m}$. c Representative Western blots and $\mathbf{d}$ densitometric analyses of levels of beclin-1 ( $n=6)$. The bars represent the mean $\pm \mathrm{SD}$. ${ }^{*} P<0.05$ vs sham control, ${ }^{\#} P<0.05$ vs vehicle control 


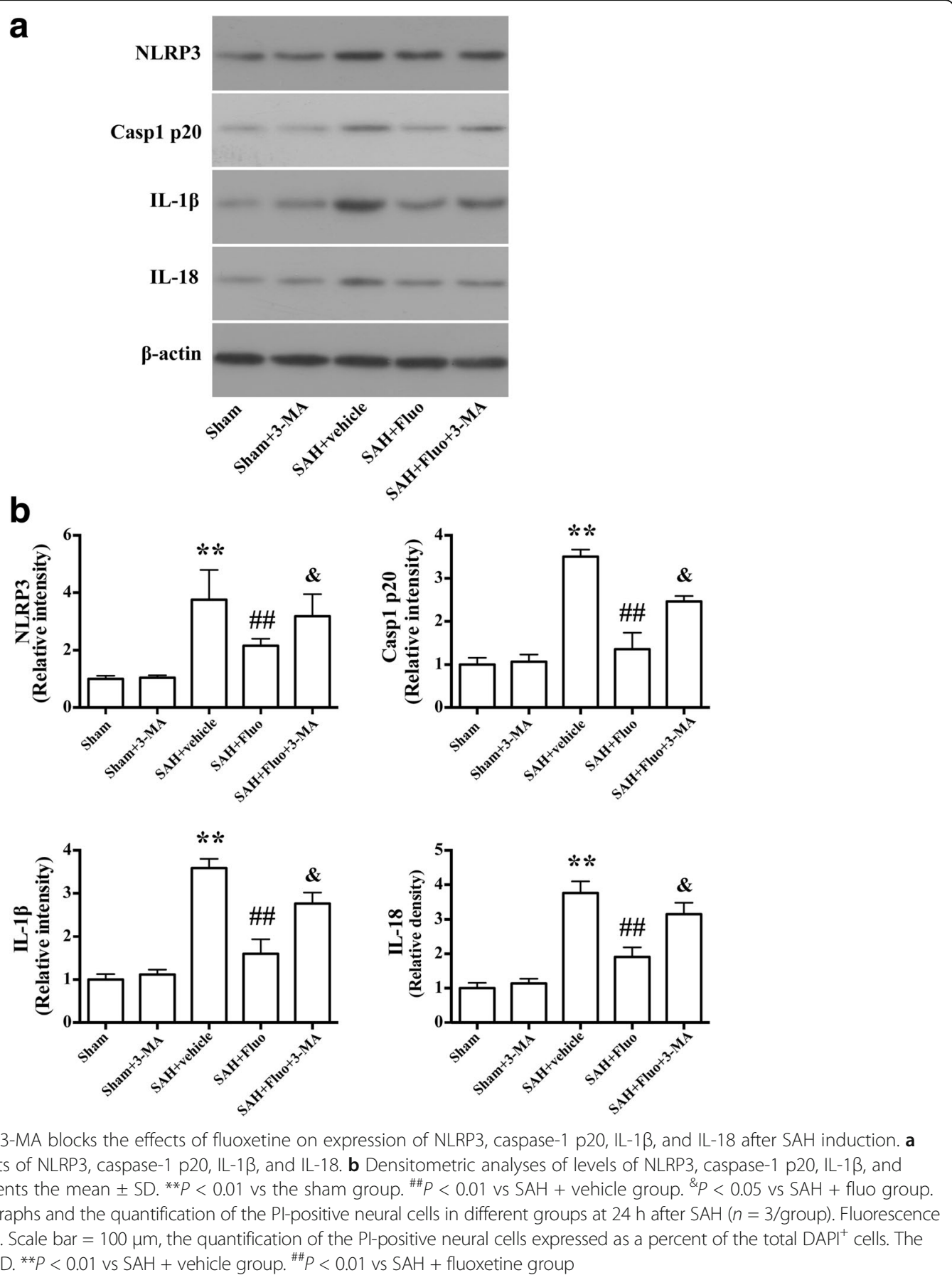

beclin-1, which is compatible with previous foundings that fluoxetine activates autophagic pathways via FKBP51 [21]. Moreover, autophagy inhibitor 3-MA reversed the neuroprotective effects of fluoxetine in our present study. These data suggest that fluoxetine provides potential therapeutic interventions for EBI after $\mathrm{SAH}$ through the inhibition of NLRP3 inflammasome activation by enhancing autophagy.
It is important to note that there are several limitations in our present study. First, the antiapoptotic and antioxidant property of fluoxetine has been described; therefore, we cannot exclude the possibility that these properties also play a role in the neuroprotective effect of fluoxetine. Second, our study showed that caspase-1 activation induced neural necrotic cell death, but whether these necrotic cell death are pyroptosis needs 


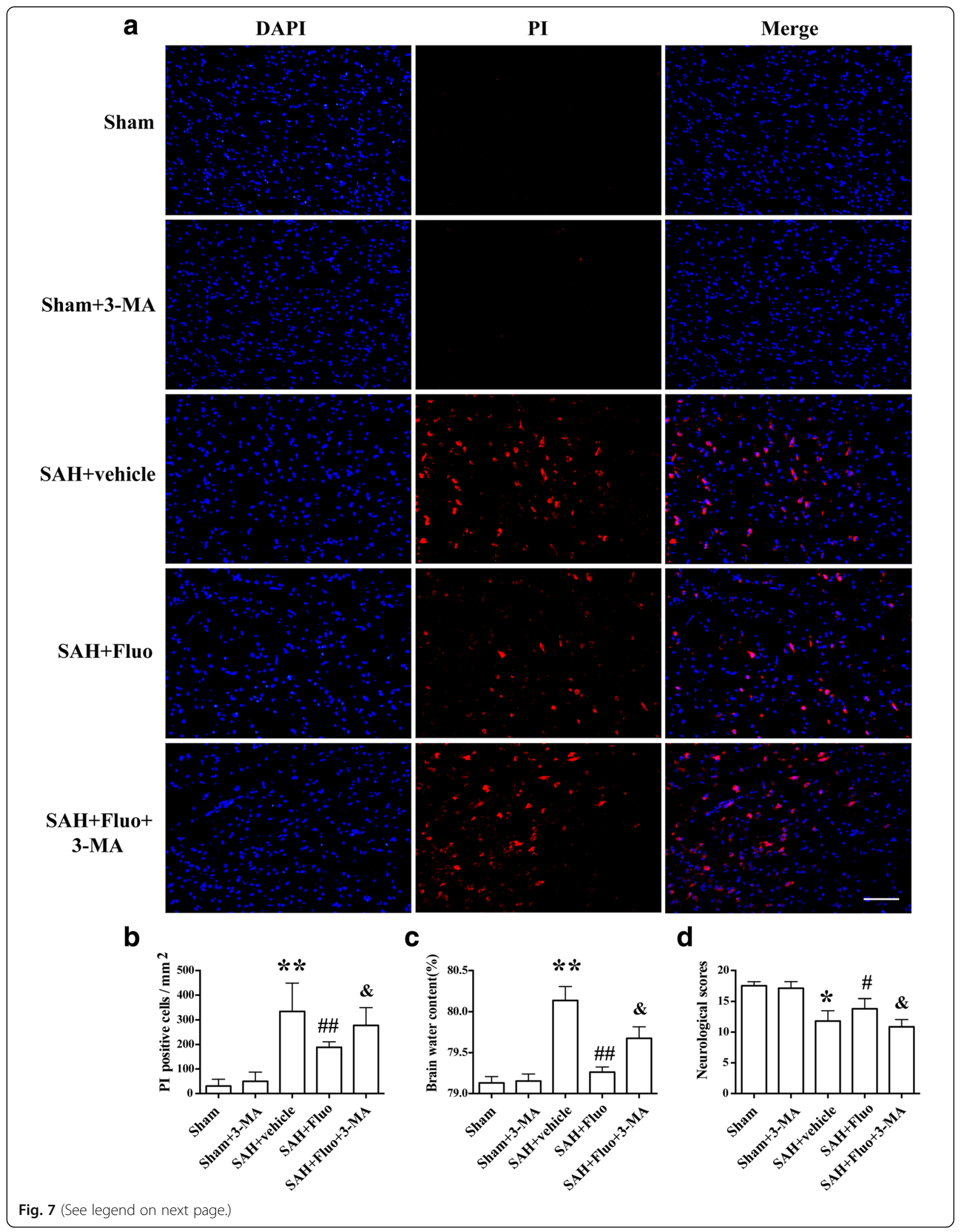


(See figure on previous page.)

Fig. 7 Autophagy inhibitor 3-MA abolishes the neuroprotective effects of fluoxetine on necrotic cell death, brain edema, and neurological function after SAH. a Representative photomicrographs of the PI-positive neural cells in different groups at $24 \mathrm{~h}$ after SAH ( $n=3 /$ group). Fluorescence colors: DAPI: blue and PI: red. Scale bar $=100 \mu \mathrm{m}$. b The quantification of the PI-positive neural cells expressed as positive cells per square millimeter. ${ }^{*} P<0.01$ vs the sham group. ${ }^{\#} P<0.01$ vs SAH + vehicle group. ${ }^{\&} P<0.05$ vs SAH + fluo group. c Quantification of brain water content $(n=6)$. The bars represent the mean \pm SD. ${ }^{* *} P<0.01$ vs the sham group. ${ }^{\# \#} P<0.01$ vs $S A H+$ vehicle group. ${ }^{\&} P<0.05$ vs SAH + fluo group. $\mathbf{d}$ Quantification of neurological scores ( $n=15$ /group). The bars represent the mean with interquartile range. ${ }^{*} P<0.05$ vs the sham group. ${ }^{\#} P<0.05$ vs $S A H+$ vehicle group. ${ }^{\&} P<0.05$ vs SAH + fluo group

further study. In addition, we studied the short-term effects of fluoxetine after SAH, but the side-effects of fluoxetine, such as myeloid response, warrant further study.

\section{Conclusion}

The present study demonstrated that NLRP3 inflammasome and caspase- 1 activation contributed to early brain injury after experimental SAH. Fluoxetine inhibits NLRP3 inflammasome activation and subsequent caspase- 1 activation and reduces early brain injury after $\mathrm{SAH}$, at least partly, by activating autophagy, providing potential therapeutic interventions for SAH.

\section{Additional files}

Additional file 1: Figure S1. The study design. (TIFF $1720 \mathrm{~kb}$ )

Additional file 2: Figure S2. Western blot figure that shows the caspase-1 antibody used in the IHC did not detect the pro-form of caspase-1. (TIFF 2497 kb)

\section{Abbreviations}

3-MA: 3-Methyladenine; Ac-YVAD-CMK: N-Ac-Tyr-Val-Ala-Asp-chloromethyl ketone; BBB: Blood brain barrier; CBF: Cerebral blood flow; CCP: Cerebral perfusion pressure; CSF: Cerebrospinal fluid; ECA: External carotid artery; FKBP51: FK506 binding protein 51; GSDMD: Gasdermin-D; ICA: Internal carotid artery; ICP: Intracranial pressure; MMP: Matrix metalloproteinase; SAH: Subarachnoid hemorrhage; SD: Sprague-Dawley; SSRI: Selective serotonin reuptake inhibitor

\section{Acknowledgements}

Not applicable

\section{Funding}

This study was supported by the National Natural Science Foundation of China (81571106 and 81601003), Natural Science Foundation of Zhejiang province (LY14H090008, LY17H090008, and LY17H090007), Project of Medicine and Health of Zhejiang Province (2014KYA093), and Province and Ministry grant (WKJZJ1517).

\section{Availability of data and materials}

All raw data used in this manuscript are available on reasonable request.

\section{Authors' contributions}

SN, YCP, FLF, ZJW, and CW performed the SAH model and Western blots. FY, $C G$, and $C W$ prepared the figures. JRL and HZX performed the immunostaining. JSC and LW performed data analysis. JRL and GC designed experiments, and JRL, HZX, and GC contributed to the writing and editing of the manuscript. All authors read and approved the manuscript.

\section{Ethics approval}

All animal experiments were approved by the Institutional Animal Care and Use Committee of Zhejiang University.
Consent for publication

Not applicable

\section{Competing interests}

The authors declare that they have no competing interests.

\section{Publisher's Note}

Springer Nature remains neutral with regard to jurisdictional claims in published maps and institutional affiliations.

\section{Author details}

'Department of Neurosurgery, The Second Affiliated Hospital of Zhejiang University School of Medicine, Hangzhou, Zhejiang, China. ${ }^{2}$ Department of Neurosurgery, Sir Run Run Shaw Hospital, Zhejiang University School of Medicine, Hangzhou, Zhejiang, China.

Received: 19 March 2017 Accepted: 5 September 2017

Published online: 13 September 2017

\section{References}

1. Connolly ES Jr, Rabinstein AA, Carhuapoma JR, Derdeyn CP, Dion J, Higashida RT, Hoh BL, Kirkness CJ, Naidech AM, Ogilvy CS, et al. Guidelines for the management of aneurysmal subarachnoid hemorrhage: a quideline for healthcare professionals from the American Heart Association/american Stroke Association. Stroke. 2012:43:1711-37.

2. Nieuwkamp DJ, Setz LE, Algra A, Linn FH, de Rooij NK, Rinkel GJ. Changes in case fatality of aneurysmal subarachnoid haemorrhage over time, according to age, sex, and region: a meta-analysis. Lancet Neurol. 2009;8:635-42.

3. Macdonald RL, Higashida RT, Keller E, Mayer SA, Molyneux A, Raabe A, Vajkoczy P, Wanke I, Bach D, Frey A, et al. Clazosentan, an endothelin receptor antagonist, in patients with aneurysmal subarachnoid haemorrhage undergoing surgical clipping: a randomised, double-blind, placebo-controlled phase 3 trial (CONSCIOUS-2). Lancet Neurol. 2011;10:618-25.

4. Macdonald RL, Higashida RT, Keller E, Mayer SA, Molyneux A, Raabe A, Vajkoczy P, Wanke I, Bach D, Frey A, et al. Randomized trial of clazosentan in patients with aneurysmal subarachnoid hemorrhage undergoing endovascular coiling. Stroke. 2012;43:1463-9.

5. Chen S, Feng H, Sherchan P, Klebe D, Zhao G, Sun X, Zhang J, Tang J, Zhang JH. Controversies and evolving new mechanisms in subarachnoid hemorrhage. Prog Neurobiol. 2014;115:64-91.

6. Sehba FA, Friedrich V. Early events after aneurysmal subarachnoid hemorrhage. Acta Neurochir Suppl. 2015;120:23-8.

7. Guo H, Callaway JB, Ting JP. Inflammasomes: mechanism of action, role in disease, and therapeutics. Nat Med. 2015;21:677-87.

8. Barrington J, Lemarchand E, Allan SM. A brain in flame; do inflammasomes and pyroptosis influence stroke pathology? Brain Pathol. 2017;27:205-12.

9. Li J, Chen J, Mo H, Chen J, Qian C, Yan F, Gu C, Hu Q, Wang L, Chen G. Minocycline protects against NLRP3 inflammasome-induced inflammation and P53-associated apoptosis in early brain injury after subarachnoid hemorrhage. Mol Neurobiol. 2016;53:2668-78.

10. Dong Y, Fan C, Hu W, Jiang S, Ma Z, Yan X, Deng C, Di S, Xin Z, Wu G, et al. Melatonin attenuated early brain injury induced by subarachnoid hemorrhage via regulating NLRP3 inflammasome and apoptosis signaling. J Pineal Res. 2016;60:253-62

11. de Rivero Vaccari JP, Dietrich WD, Keane RW. Activation and regulation of cellular inflammasomes: gaps in our knowledge for central nervous system injury. J Cereb Blood Flow Metab. 2014;34:369-75.

12. de Vasconcelos NM, Van Opdenbosch N, Lamkanfi M. Inflammasomes as polyvalent cell death platforms. Cell Mol Life Sci. 2016;73:2335-47. 
13. Shao A, Wu H, Hong Y, Tu S, Sun X, Wu Q, Zhao Q, Zhang J, Sheng J. Hydrogen-rich saline attenuated subarachnoid hemorrhage-induced early brain injury in rats by suppressing inflammatory response: possible involvement of NF-kappaB pathway and NLRP3 inflammasome. Mol Neurobiol. 2016;53:3462-76.

14. Cao S, Shrestha S, Li J, Yu X, Chen J, Yan F, Ying G, Gu C, Wang L, Chen G. Melatonin-mediated mitophagy protects against early brain injury after subarachnoid hemorrhage through inhibition of NLRP3 inflammasome activation. Sci Rep. 2017;7:2417.

15. Harris J, Lang T, Thomas JPW, Sukkar MB, Nabar NR, Kehrl JH. Autophagy and inflammasomes. Mol Immunol. 2017;86:10-5.

16. Martins JD, Liberal J, Silva A, Ferreira I, Neves BM, Cruz MT. Autophagy and inflammasome interplay. DNA Cell Biol. 2015;34:274-81.

17. Wong DT, Perry KW, Bymaster FP. Case history: the discovery of fluoxetine hydrochloride (Prozac). Nat Rev Drug Discov. 2005:4:764-74.

18. Chollet F, Tardy J, Albucher JF, Thalamas C, Berard E, Lamy C, Bejot Y, Deltour $\mathrm{S}$, Jaillard $\mathrm{A}$, Niclot $\mathrm{P}$, et al. Fluoxetine for motor recovery after acute ischaemic stroke (FLAME): a randomised placebo-controlled trial. Lancet Neurol. 2011;10:123-30.

19. McAllister BB, Spanswick SC, Patel PP, Barneto AA, Dyck RH. The effects of chronic fluoxetine treatment following injury of medial frontal cortex in mice. Behav Brain Res. 2015;290:102-16.

20. Lee JY, Kim HS, Choi HY, Oh TH, Yune TY. Fluoxetine inhibits matrix metalloprotease activation and prevents disruption of blood-spinal cord barrier after spinal cord injury. Brain. 2012;135:2375-89.

21. Gassen NC, Hartmann J, Zschocke J, Stepan J, Hafner K, Zellner A, Kirmeier T, Kollmannsberger L, Wagner KV, Dedic N, et al. Association of FKBP51 with priming of autophagy pathways and mediation of antidepressant treatment response: evidence in cells, mice, and humans. PLoS Med. 2014;11: e1001755.

22. Du RH, Tan J, Sun XY, Lu M, Ding JH, Hu G. Fluoxetine inhibits NLRP3 inflammasome activation: implication in depression. Int J Neuropsychopharmacol. 2016;19:pyw037.

23. Ying GY, Jing $\mathrm{CH}$, Li JR, Wu C, Yan F, Chen JY, Wang L, Dixon BJ, Chen G. Neuroprotective effects of valproic acid on blood-brain barrier disruption and apoptosis-related early brain injury in rats subjected to subarachnoid hemorrhage are modulated by heat shock protein 70/matrix metalloproteinases and heat shock protein 70/AKT pathways. Neurosurgery. 2016;79:286-95

24. Sozen T, Tsuchiyama R, Hasegawa Y, Suzuki H, Jadhav V, Nishizawa S, Zhang $J$ H. Role of interleukin-1beta in early brain injury after subarachnoid hemorrhage in mice. Stroke. 2009;40:2519-25.

25. Lim CM, Kim SW, Park JY, Kim C, Yoon SH, Lee JK. Fluoxetine affords robust neuroprotection in the postischemic brain via its anti-inflammatory effect. J Neurosci Res. 2009;87:1037-45.

26. Jing $\mathrm{CH}$, Wang L, Liu PP, Wu C, Ruan D, Chen G. Autophagy activation is associated with neuroprotection against apoptosis via a mitochondria pathway in a rat model of subarachnoid hemorrhage. Neuroscience. 2012; 213:144-53.

27. Sugawara $T$, Ayer $R$, Jadhav $V$, Zhang JH. A new grading system evaluating bleeding scale in filament perforation subarachnoid hemorrhage rat model. J Neurosci Methods. 2008;167:327-34

28. Chen J, Chen G, Li J, Qian C, Mo H, Gu C, Yan F, Yan W, Wang L. Melatonin attenuates inflammatory response-induced brain edema in early brain injury following a subarachnoid hemorrhage: a possible role for the regulation of pro-inflammatory cytokines. J Pineal Res. 2014;57:340-7.

29. Takeuchi O, Akira S. Pattern recognition receptors and inflammation. Cell. 2010;140:805-20.

30. Wu Q, Wang XL, Yu Q, Pan H, Zhang XS, Zhang QR, Wang HD, Zhang X. Inflammasome proteins in cerebrospinal fluid of patients with subarachnoid hemorrhage are biomarkers of early brain injury and functional outcome. World Neurosurg. 2016;94:472-9.

31. Hendryk S, Jarzab B, Josko J. Increase of the IL-1 beta and IL-6 levels in CSF in patients with vasospasm following aneurysmal SAH. Neuro Endocrinol Lett. 2004;25:141-7.

32. Ding J, Wang K, Liu W, She Y, Sun Q, Shi J, Sun H, Wang DC, Shao F. Poreforming activity and structural autoinhibition of the gasdermin family. Nature. 2016;535:111-6.

33. Shi J, Zhao $Y$, Wang $K$, Shi $X$, Wang $Y$, Huang $H$, Zhuang $Y$, Cai T, Wang F, Shao F. Cleavage of GSDMD by inflammatory caspases determines pyroptotic cell death. Nature. 2015;526:660-5.
34. Wallach D, Kang TB, Dillon CP, Green DR. Programmed necrosis in inflammation: toward identification of the effector molecules. Science. 2016;352:aaf2154.

35. Tan MS, Tan L, Jiang T, Zhu XC, Wang HF, Jia CD, Yu JT. Amyloid-beta induces NLRP1-dependent neuronal pyroptosis in models of Alzheimer's disease. Cell Death Dis. 2014;5:e1382.

36. Brennan MA, Cookson BT. Salmonella induces macrophage death by caspase-1-dependent necrosis. Mol Microbiol. 2000;38:31-40.

37. Chen X, He WT, Hu L, Li J, Fang Y, Wang X, Xu X, Wang Z, Huang K, Han J. Pyroptosis is driven by non-selective gasdermin-D pore and its morphology is different from MLKL channel-mediated necroptosis. Cell Res. 2016;26: 1007-20.

38. Amin J, Boche D, Rakic S. What do we know about the inflammasome in humans? Brain Pathol. 2017;27:192-204

39. Liu H, Zhao L, Yue L, Wang B, Li X, Guo H, Ma Y, Yao C, Gao L, Deng J, et al. Pterostilbene attenuates early brain injury following subarachnoid hemorrhage via inhibition of the NLRP3 inflammasome and Nox2-related oxidative stress. Mol Neurobiol. 2017;54:5928-40.

40. Mowla A, Mosavinasab M, Pani A. Does fluoxetine have any effect on the cognition of patients with mild cognitive impairment? A double-blind, placebo-controlled, clinical trial. J Clin Psychopharmacol. 2007;27:67-70.

41. Horsfield SA, Rosse RB, Tomasino V, Schwartz BL, Mastropaolo J, Deutsch SI. Fluoxetine's effects on cognitive performance in patients with traumatic brain injury. Int J Psychiatry Med. 2002;32:337-44.

42. Su HC, Ma CT, Yu BC, Chien YC, Tsai CC, Huang WC, Lin CF, Chuang YH, Young KC, Wang JN, Tsao CW. Glycogen synthase kinase-3beta regulates antiinflammatory property of fluoxetine. Int Immunopharmacol. 2012;14:150-6.

43. Alboni S, Poggini S, Garofalo S, Milior G, El Hajj H, Lecours C, Girard I, Gagnon S, Boisjoly-Villeneuve S, Brunello N, et al. Fluoxetine treatment affects the inflammatory response and microglial function according to the quality of the living environment. Brain Behav Immun. 2016;58:261-71.

44. Glick D, Barth S, Macleod KF. Autophagy: cellular and molecular mechanisms. J Pathol. 2010:221:3-12.

45. Lee JY, He Y, Sagher O, Keep R, Hua Y, Xi G. Activated autophagy pathway in experimental subarachnoid hemorrhage. Brain Res. 2009;1287:126-35.

46. Wang Z, Shi XY, Yin J, Zuo G, Zhang J, Chen G. Role of autophagy in early brain injury after experimental subarachnoid hemorrhage. J Mol Neurosci. 2012;46:192-202.

47. Saitoh T, Fujita N, Jang MH, Uematsu S, Yang BG, Satoh T, Omori H, Noda T, Yamamoto N, Komatsu M, et al. Loss of the autophagy protein Atg16L1 enhances endotoxin-induced IL-1 beta production. Nature. 2008;456:264-8.

48. Shibutani ST, Saitoh T, Nowag H, Munz C, Yoshimori T. Autophagy and autophagyrelated proteins in the immune system. Nat Immunol. 2015;16:1014-24.

49. Ding Z, Liu S, Wang X, Dai Y, Khaidakov M, Deng X, Fan Y, Xiang D, Mehta JL. LOX-1, mtDNA damage, and NLRP3 inflammasome activation in macrophages: implications in atherogenesis. Cardiovasc Res. 2014;103:619-28.

50. Nakahira K, Haspel JA, Rathinam VA, Lee SJ, Dolinay T, Lam HC, Englert JA, Rabinovitch M, Cernadas M, Kim HP, et al. Autophagy proteins regulate innate immune responses by inhibiting the release of mitochondrial DNA mediated by the NALP3 inflammasome. Nat Immunol. 2011;12:222-30.

51. Harris J, Hartman M, Roche C, Zeng SG, O'Shea A, Sharp FA, Lambe EM, Creagh EM, Golenbock DT, Tschopp J, et al. Autophagy controls IL-1 beta secretion by targeting pro-IL-1 beta for degradation. J Biol Chem. 2011;286:9587-97.

52. Shi CS, Shenderov K, Huang NN, Kabat J, Abu-Asab M, Fitzgerald KA, Sher A, Kehrl JH. Activation of autophagy by inflammatory signals limits IL-1beta production by targeting ubiquitinated inflammasomes for destruction. Nat Immunol. 2012;13:255-63. 